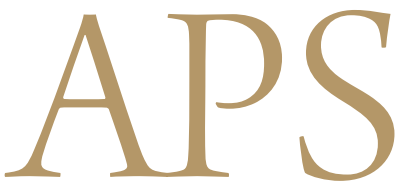

Archives of Plastic Surgery

\title{
Bio-Conjugated Polycaprolactone Membranes: A Novel Wound Dressing
}

\author{
Elijah Zhengyang $\mathrm{Cai}^{1 *}$, Erin Yiling Teo ${ }^{2 *}$, Lim Jing ${ }^{3}$, Yun Pei $\mathrm{Koh}^{1}$, Tan Si Qian ${ }^{4}$, Feng Wen ${ }^{3}$, \\ James Wai Kit Lee ${ }^{1}$, Eileen Chor Hoong Hing ${ }^{5,6}$, Yan Lin Yap ${ }^{6}$, Hanjing Lee ${ }^{6}$, \\ Chuen Neng Lee ${ }^{5,7}$, Swee-Hin Teoh ${ }^{3}$, Jane Lim ${ }^{5,6}$, Thiam Chye Lim ${ }^{5,6}$ \\ ${ }^{1}$ Department of Surgery, National University Health System, Singapore; ${ }^{2}$ Department of Reproductive Medicine, KK Women's and Children \\ Hospital, Singapore; ${ }^{3}$ Division of Bioengineering, School of Chemical and Biomedical Engineering, Nanyang Technological University, \\ Singapore; ${ }^{4}$ Centre for Biomedical Materials Applications and Technology (BIOMAT), Department of Mechanical Engineering, Faculty of \\ Engineering, National University of Singapore, Singapore; ${ }^{5}$ Department of Surgery, National University of Singapore, Singapore; ${ }^{6}$ Division of \\ Plastic, Reconstructive and Aesthetic Surgery, Department of Surgery, National University Health System, Singapore; ${ }^{7}$ Division of Cardiac, \\ Thoracic and Vascular Surgery, Department of Surgery, National University Health System, Singapore
}

Background The combination of polycaprolactone and hyaluronic acid creates an ideal environment for wound healing. Hyaluronic acid maintains a moist wound environment and accelerates the in-growth of granulation tissue. Polycaprolactone has excellent mechanical strength, limits inflammation and is biocompatible. This study evaluates the safety and efficacy of bio-conjugated polycaprolactone membranes (BPM) as a wound dressing.

Methods $16 \mathrm{New}$ Zealand white rabbits were sedated and local anaesthesia was administered. Two $3.0 \times 3.0 \mathrm{~cm}$ full-thickness wounds were created on the dorsum of each rabbit, between the lowest rib and the pelvic bone. The wounds were dressed with either BPM $(n=12)$ or Mepitel $(n=12)$ (control), a polyamide-silicon wound dressing. These were evaluated macroscopically on the 7th, 14th, 21st, and 28th postoperative days for granulation, reepithelialization, infection, and wound size, and histologically for epidermal and dermal regeneration.

Results Both groups showed a comparable extent of granulation and re-epithelialization. No signs of infection were observed. There was no significant difference $(P>0.05)$ in wound size between the two groups. BPM $(\mathrm{n}=6): 8.33 \mathrm{~cm}^{2}, 4.90 \mathrm{~cm}^{2}, 3.12 \mathrm{~cm}^{2}, 1.84 \mathrm{~cm}^{2}$; Mepitel $(\mathrm{n}=6)$ : $10.29 \mathrm{~cm}^{2}, 5.53 \mathrm{~cm}^{2}, 3.63 \mathrm{~cm}^{2}, 2.02 \mathrm{~cm}^{2}$; at the 7th, 14th, 21st, and 28th postoperative days. The extents of epidermal and dermal regeneration were comparable between the two groups. Conclusions BPM is comparable to Mepitel as a safe and efficacious wound dressing.

Keywords Skin / Wounds / Hyaluronic acid / Polycaprolactone / Rabbit
Correspondence: Thiam Chye Lim Department of Surgery (Division of Plastic, Aesthetic and Reconstructive Surgery), Yong Loo Lin School of Medicine, National University of Singapore, NUHS Tower Block, Level 8, 1E Kent Ridge Road, Singapore 119228, Singapore Tel: +65-6772-2022

Fax: +65-6777-8427

E-mail: thiam_chye_lim@nuhs.edu.sg

*These authors contributed equally to this work.

This work is funded by the Ministry of Education, Academic Research Fund (Tier 1), R265-000-343-112.

This article was presented (oral presentation) during the 3rd World Congress for Plastic Surgeons of Chinese Descent, Xi An, China on October 2012.

We would like to acknowledge the following organizations for providing the facilities and resources necessary for conducting the study: 1 . Centre for Biomedical Materials Applications and Technology (BIOMAT), Department of Mechanical Engineering, Faculty of Engineering, National University of Singapore, Singapore 2. Osteopore International Pte Ltd, Singapore.

No potential conflict of interest relevant to this article was reported. 


\section{INTRODUCTION}

An ideal wound dressing would allow adequate gaseous exchange, maintain a moist environment while preventing maceration, allow drainage and absorption of exudates, protect the wound from trauma and infection, facilitate debridement, minimize scarring, and be non-adherent during dressing changes, minimizing trauma and pain. Other practical considerations include cost, availability and stability in storage and use [1].

Biopolymers have been investigated for their potential as a primary wound dressing. Key requirements include biocompatibility and the lack of antigenicity. Polycaprolactone (PCL) has been utilized as an ultra-thin film for dressing cutaneous wounds [2]. PCL possesses several advantages over other polymers including stability in ambient conditions, low cost, and ready availability in large quantities. It has the advantages of having good mechanical strength and the ability to minimize inflammation, and has been proven to be biocompatible [3].

Hyaluronic acid (HyA) has the ability to minimize inflammatory response and maintain a moist wound environment, which plays a major role in promoting wound healing [4]. Poor mechanical strength limits its applications. We have developed a new method of hyaluronic acid engraftment onto PCL. PCL was chosen as a scaffold for surface conjugation in view of its excellent mechanical strength and its inert properties as noted above. This study evaluates the safety and efficacy of our novel bio-conjugated PCL membrane as a wound dressing.

\section{METHODS}

All reagents were obtained from Sigma-Aldrich Singapore un- less otherwise stated.

\section{Fabrication of bi-axially stretched PCL membrane}

The fabrication of the bi-axially stretched medical grade PCL (mPCL) membrane was as described by Tiaw et al. [5]. Briefly, medical grade PCL pellets (Osteopore International Pte Ltd, Singapore) were put through 2 heated roll mills and mixed into mPCL masses. The mPCL masses were then heat pressed at high temperature and pressure to obtain a membrane thickness of $100-150 \mu \mathrm{m}$. The mPCL membranes were lastly bi-axially stretched to a membrane thickness of $10-30 \mu \mathrm{m}$ with a $3 \times 3$ draw ratio and cut into pieces of $3 \mathrm{~cm} \times 1 \mathrm{~cm}$.

\section{Surface modification and conjugation of hyaluronic acid onto membrane}

Pre-treatment of the MPCL membranes was first carried out by subjecting them to radio frequency glow-discharge argon plasma (PX250; March Systems, Nordson Corp., Westlake, OH, USA). The parameters were fixed at $100 \mathrm{~W}$ for $20 \mathrm{~s}$ of radio-frequency glow discharge time and then $3 \mathrm{~min}$ of exposure to air to achieve optimal hydrophilicity with the formation of peroxides. $50 \% \mathrm{w} / \mathrm{v}$ polyethyleneimine (PEI) dissolved in industrial ethanol $(50 \% \mathrm{w} /$ $\mathrm{v}$ PEI) was spin-coated onto the $\mathrm{mPCL}$ membrane at 2,460 rpm, after which, the adsorbed PEI was cross-linked onto the MPCL membrane using similar plasma crosslinking (Fig. 1). Any amounts of PEI that were not cross-linked onto the surface were washed and removed by immersion in de-ionized water overnight with agitation.

Surface conjugation of hyaluronic acid (HyA) was then carried out by first dissolving $0.1 \mathrm{w} / \mathrm{w} \%$ bio-sodium hyaluronate $(\mathrm{Mw}=$ $2.0 \times 10^{6} \mathrm{Da}$; Shiseido Co. Ltd., Tokyo, Japan) in a combined solu-

\section{Fig. 1. Mechanism for immobilizing HyA in PEI/HyA-mPCL}

PEI dissolved in ethanol was spin coated onto the $\mathrm{mPCL}$ membrane. The adsorbed PEI were crossedlinked onto the $\mathrm{MPCL}$ membrane. HyA, hyaluronic acid; PEI/HyA-mPCL, bioconjugated polycaprolactone membrane; PEl, polyethyleneimine; $\mathrm{mPCL}$, medical grade polycaprolactone.

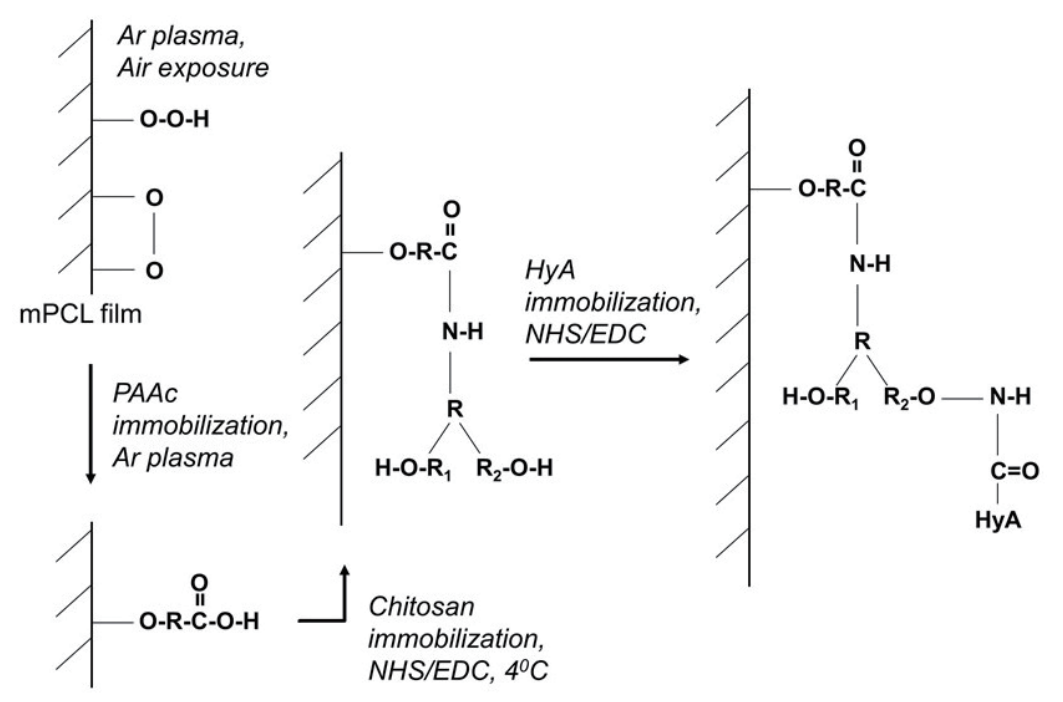


tion of MES buffer ( $\mathrm{pH}$ 5.5) and N-hydroxysuccinimide/1-ethyl3-(3-dimethylaminopropyl carbodiimide hydrochloride solution (NHS/EDC). The combined solution of MES buffer and NHS/ EDC solution was prepared by adding $60 \mathrm{mg}$ of EDC (from Fluka Analytical, Sigma-Aldrich, Singapore, Singapore) and $36 \mathrm{mg}$ of NHS powder into $3 \mathrm{~mL}$ of $0.05 \mathrm{M} \mathrm{MES}$ buffer ( $\mathrm{pH}$ 5.5). The final solution was then used to immerse the PEI-cross-linked mPCL membrane at room temperature. Any excess HyA was then removed by immersion in de-ionised water for an hour.

\section{In vitro analysis}

FTIR-ATR

Fourier transform infrared-attenuated total reflectance (FTIRATR) was another method used to identify the types of functional groups present on the surface of $\mathrm{MPCL}$ membranes. The membranes were placed on an ATR crystal for surface chemical analysis using the FTIR-ATR setup (Avatar 360 FT-IR) (Thermo Fisher Scientific Inc., Waltham, MA, USA).

\section{X-ray photoelectron spectroscopy (XPS)}

The surface elements present on the HyA-grafted MPCL membranes were analysed and evaluated with a Mono Al Kalpha XPS AXIS UltraDLD (Kratos, USA) (set at $1486.71 \mathrm{eV}, 5 \mathrm{~mA}$, $15 \mathrm{kV}, 75 \mathrm{~W}$ ). A wide scan survey spectrum was obtained and surface elemental stoichiometries were determined from peakarea ratios as described in Chong et al. [6].

\section{Cytocompatibility assay}

The cytocompatibility of hyaluronic acid grafted mPCL (HyAmPCL) was evaluated according to ISO 10993 guidelines. Briefly, $8 \times 10^{4}$ human dermal fibroblast cells cultured in a standard incubator with Dulbecco's modified Eagle’s medium (Gibco, Life Technologies, Carlsbad, CA, USA), supplemented with 10\% foetal bovine serum (DMEM-FBS) were seeded onto each specimen of $12 \mathrm{~mm}$ in diameter. The cells were cultured for 28 days and evaluated periodically (day 1, 3, 7, 21, 28) for cell viability using the metabolic Alarmar blue assay. Glass slides of $12 \mathrm{~mm}$ in diameter were used as a positive control.

\section{In vivo analysis}

Rabbit full thickness wound model

The protocol used was adapted from Teo et al. [2]. New Zealand White rabbits $(2-2.5 \mathrm{~kg})$ were used in this study. The rabbits were housed individually. All rabbits were acclimatized for one week before the surgery. All handling, maintenance and protocols were in accordance to our institute's Animal Care and Use Committee.

Two full thickness excisional wounds (to the panniculus adi- posus) of $3 \mathrm{~cm} \times 3 \mathrm{~cm}$ were created on the dorsum between the inferior border of the lowest rib and the superior border of the pelvic bone of each of the 16 rabbits with aseptic techniques. Wounds were created on each side of the midline at a distance of $1 \mathrm{~cm}$. We used a template to mark out the areas on un-stretched skin. Each original wound area was measured using a sterile transparent graph sheet prior to the application of wound dressing. Sterilized bio-conjugated PCL membranes (treatment) and Mepitel (Molnlycke Health Care, Gothenburg, Sweden) (control) were used for the wounds on each side respectively. Both dressings were anchored to the superior 2 corners of the wound using 5-0 nylon suture. The wounds were covered with a standard secondary dressing of gauze and stockinet.

\section{Wound contracture measurement}

The wounds were photographed with the rabbits in a standard prone position using an 8 megapixels digital camera (PowerShot A60, Canon, Melville, NY, USA). The wound area was measured by tracing the margins of the wounds onto a transparency graph film. The wound size was determined by counting the number of squares that were marked out [7]. The wound area was measured on a weekly basis, up to a maximum of 4 weeks. The percentage of wound contraction was then defined as: Percentage wound closure $=\left(A_{0}-A_{t}\right) / A_{0} \times 100$ where $A_{0}$ is the original wound area and $A t$ is the area of wound at a given point in time [8].

\section{Macroscopic evaluation}

The gross appearance of the wounds were evaluated for signs of infection, the degree of granulation, re-epithelialization and scarring [9]. Mechanical factors such as the ease of handling during dressing application and dressing change, the ability to conform to the wound surface, the migration of dressings from the wound sites, and the extent of exudate collected were also noted.

\section{Histology}

Batches of rabbits $(n=4)$ were then sacrificed at days 7, 14, 21, and 28, respectively. Excisional biopsies of the wounds were obtained and fixed in formalin (10\%). Tissue samples were embedded in paraffin, sectioned and stained with hematoxylin and eosin for general analyses and Masson's trichrome for identifying the presence of collagen in the wound tissue. Representative images were captured to facilitate evaluation of epidermal and dermal healing. A pathologist evaluated the histological data for both the treatment and control groups, and the resulting analysis was taken into consideration.

\section{Statistical analysis}

Statistical comparisons for significance were conducted using 
the Student's t-test with a 95\% confidence level. Data points were expressed as means \pm standard deviations.

\section{RESULTS}

\section{In vitro evaluation}

\section{FTIR-ATR}

The surface chemistries of the PEI/HyA-mPCL membrane were evaluated using FTIR-ATR, and the resulting graphs are shown in Fig. 2. It was shown that the graph derived from naïve mPCL was significantly different from that of the HyA-mPCL membranes with different peaks in different regions. PEI/HyAmPCL membrane showed peaks around the range of $1,700 \mathrm{~cm}^{-1}$ and $1,500 \mathrm{~cm}^{-1}$.

\section{X-ray photoelectron spectroscopy (XPS)}

The XPS survey scans of naïve $\mathrm{MPCL}$ and PEI/HyA-mPCL membranes are shown in Fig. 3. PEI/HyA-mPCL contained carbon, oxygen, and nitrogen elements. After immobilizing HyA onto $\mathrm{mPCL}$ films, corresponding peaks in nitrogen could be observed.

Fig. 4 illustrates the deconvolution of the XPS peaks. Four peaks were required for curve-fitting: the amide (CONH), hydroxyl $(-\mathrm{COH})$, saturated carbon and secondary carbon groups. The amide and hydroxyl groups were further analysed for their relative atomic percentage (at\%) within these narrow scans (Table 1). For PEI/HyA-mPCL membranes, the amide and hydroxyl groups were centred at $288.25 \mathrm{eV}$ and $286.47 \mathrm{eV}$ respectively, with $\mathrm{X}_{\mathrm{at}}$ of $16.0 \%$ and $48.3 \%$ respectively. From

\section{Fig. 2. FTIR-ATR spectra of naïve $m P C L$ and $P E I / H y A-m P C L$}

Graph derived from naïve $\mathrm{mPCL}$ was significantly different from that of the HyA-mPCL membranes with different peaks in different regions. FIR-ATR, fourier transform infrared-attenuated total reflectance; $\mathrm{mPCL}$, medical grade polycaprolactone; PEI/ HyA-mPCL, bioconjugated polycaprolactone membrane; PEI, polyethyleneimine; HyA, hyaluronic acid.
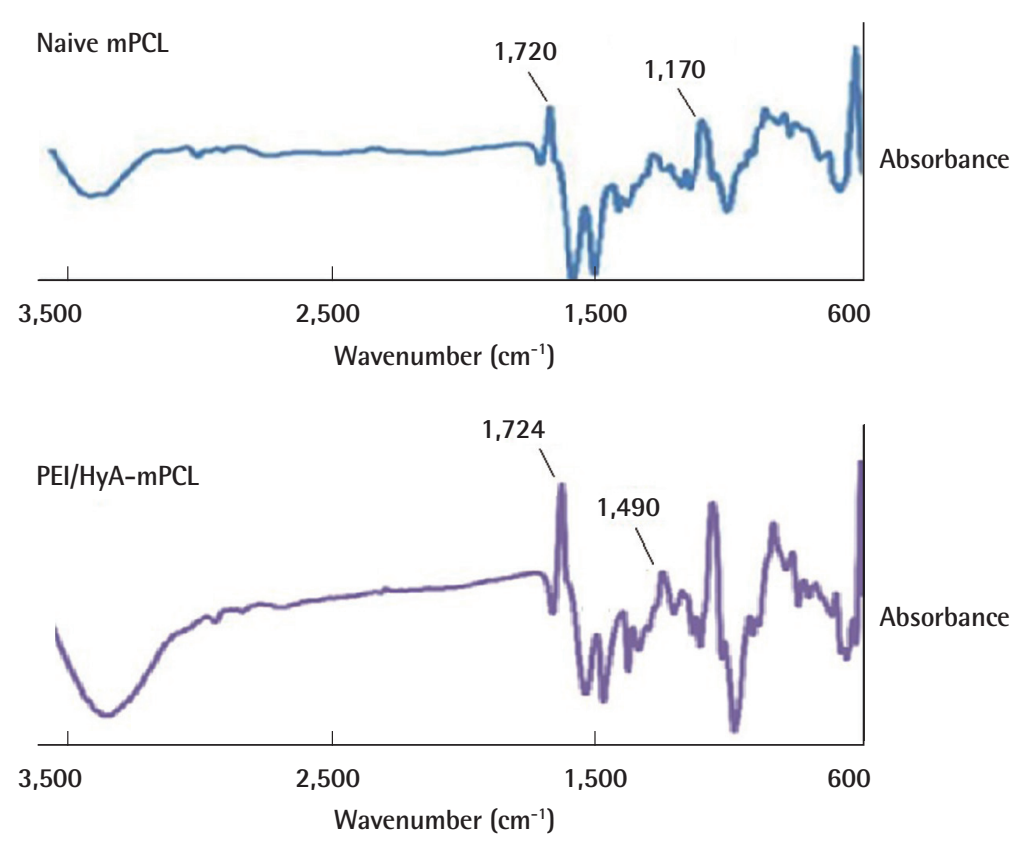

Fig. 3. XPS spectra of $m P C L$ and PEI/HyA-mPCL

Nitrogen was detected amongst the elemental composition following the engraftment of HyA. XPS, X-ray photoelectron spectroscopy; $\mathrm{mPCL}$, medical grade polycaprolactone; PEI/HyA-mPCL, bioconjugated polycaprolactone membrane.
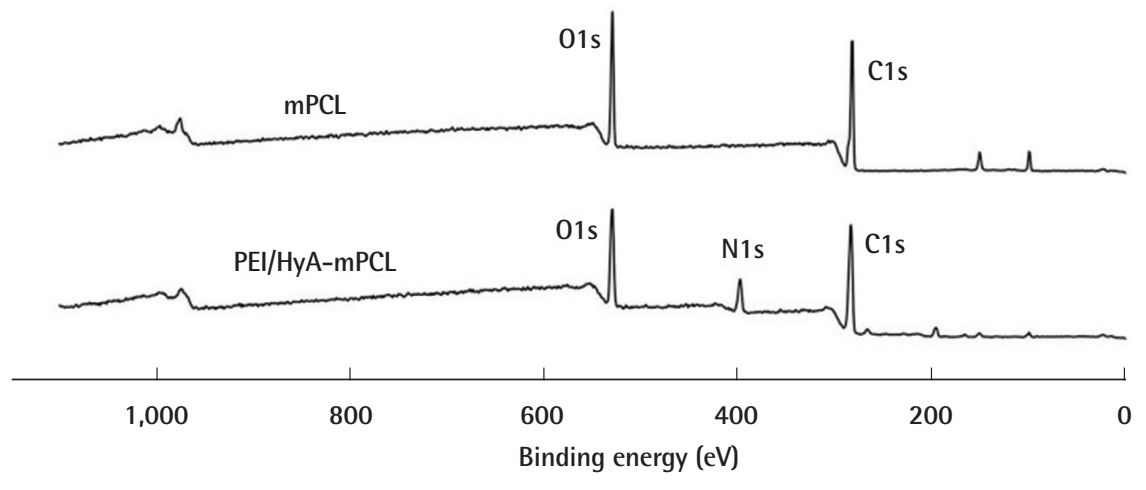


\section{Fig. 4. Narrow scan of C1s spectra of PEl/HyA-mPCL}

The amide and hydroxyl functional groups were detected, suggesting that HyA has been successfully engrafted. $\mathrm{mPCL}$, medical grade polycaprolactone; PEI/HyA-mP$\mathrm{CL}$, bioconjugated polycaprolactone membrane.

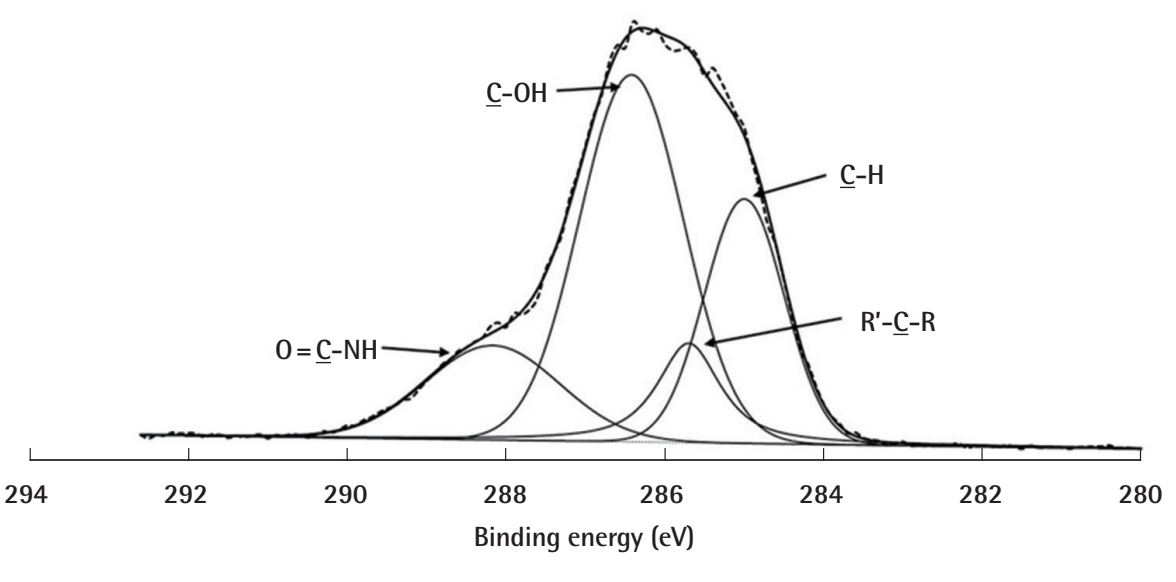

Table 1. C1s peak fit analyses (-CONH and -COH)

\begin{tabular}{|c|c|c|c|c|}
\hline \multirow{2}{*}{ Film } & \multicolumn{2}{|c|}{$-\mathrm{CONH}$} & \multicolumn{2}{|c|}{$-\mathrm{COH}$} \\
\hline & Center $(\mathrm{eV})$ & $\mathrm{X}_{\mathrm{at}}(\%)$ & Center (eV) & $\mathrm{X}_{\mathrm{at}}(\%)$ \\
\hline $\mathrm{PE} / \mathrm{HyA}-\mathrm{mPCL}$ & 288.25 & 16.0 & 286.47 & 48.3 \\
\hline \multicolumn{5}{|c|}{$\begin{array}{l}\text { The amide and hydroxyl groups were centered at } 288.25 \mathrm{eV} \text { and } 286.47 \mathrm{eV} \text { re- } \\
\text { spectively, with } \mathrm{X}_{\mathrm{at}} \text { of } 16.0 \% \text { and } 48.3 \% \text { respectively. } \\
\text {-CONH, amide group; -COH, hydroxyl group; Xat\%, relative atomic percentage; } \\
\mathrm{eV} \text {, energy loss; PEI/HyA-mPCL, bioconjugated polycaprolactone membrane. }\end{array}$} \\
\hline
\end{tabular}

\section{Fig. 5. Graph of percentage cell viability}

There is a consistently high level of cell viability throughout the 28 days of culture for hyaluronic acid grafted $\mathrm{MPCL}$ fabricated by the PEI method. $\mathrm{mPCL}$, medical grade polycaprolactone; PEI, polyethyleneimine.

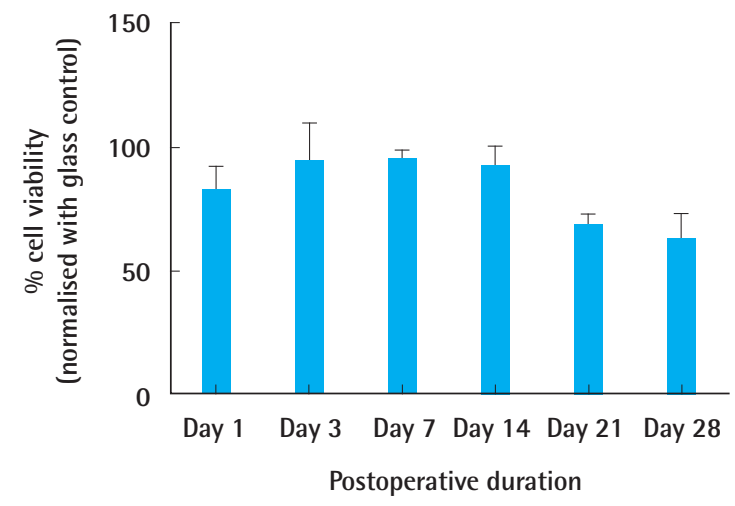

these results, it was observed that the PEI/HyA-mPCL membranes had HyA successfully immobilized on their surfaces.

\section{Cytocompatibility assay}

The cell viability when normalised to a positive control (glass slide) was as shown in Fig. 5. A general increasing trend of percentage cell viability with time was observed within the first 7 days for PEI. It was also noted that there was a consistently high
Fig. 6. Graph of wound size

A decreasing trend in wound area can been observed in both groups. PCL, polycaprolactone.

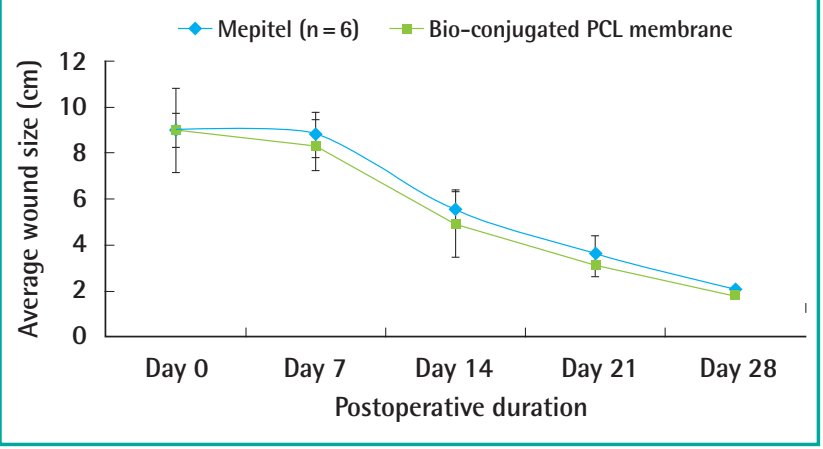

Fig. 7. Graph of percentage reduction in wound area

Wound area decreased sharply to approximately $40 \%$ of its original area by day 7 . Wounds from both groups achieved an average of $84 \%$ wound contracture by day 28. PCL, polycaprolactone.

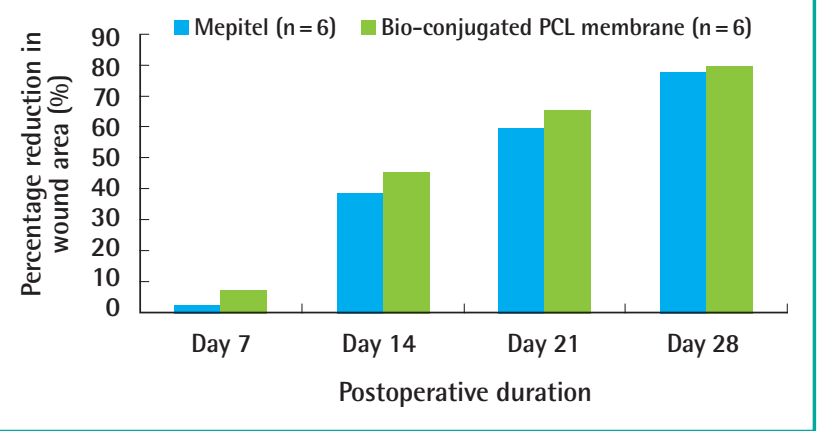

level (above $70 \%$ ) of cell viability throughout the 28 days of culture.

\section{In vivo evaluation}

Wound contracture measurement

A gradual reduction in the wound area was seen in both the treatment group (bio-conjugated PCL membrane) and the 
Fig. 8. Representative images of wounds

All the wounds showed similar extent of granulation and epithelialization. PCL, polycaprolactone.

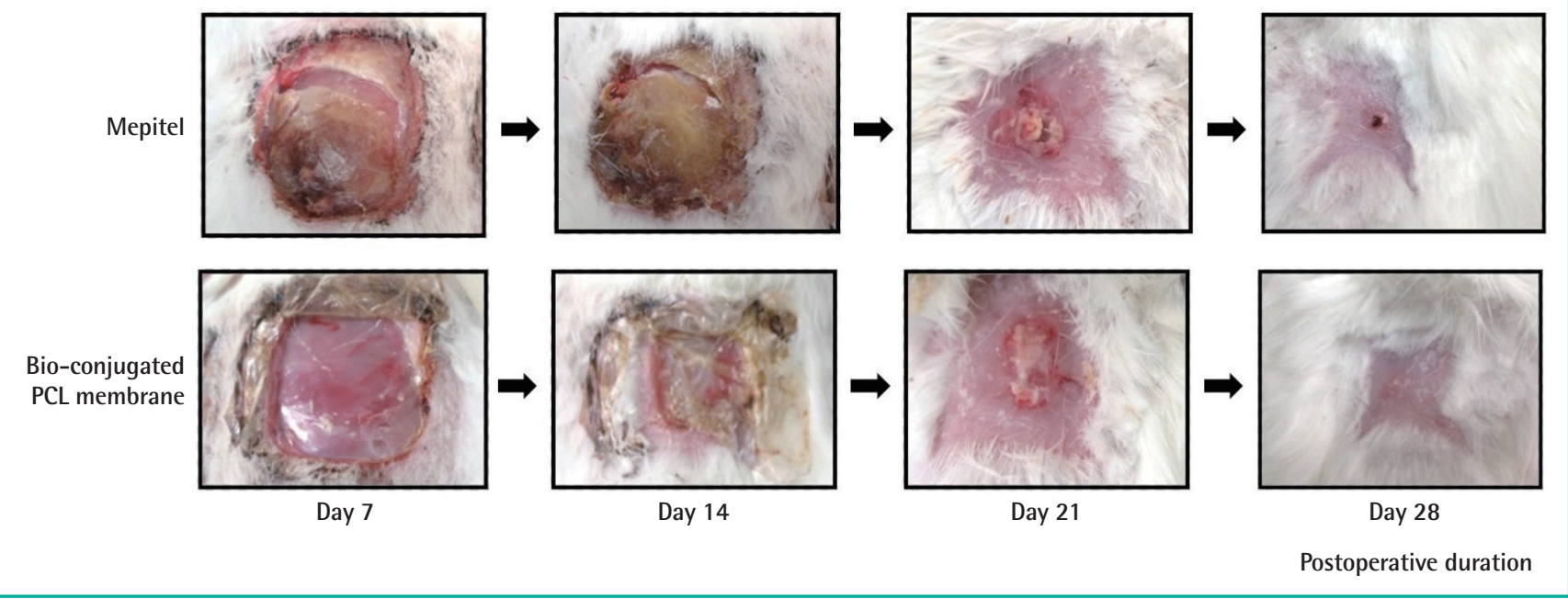

control group (Mepitel) over 28 days (Fig. 6). Throughout the study, there was no statistical difference observed between the wound size of the control group and treatment group. From day 7 onwards, the wound area decreased sharply to approximately $40 \%$ of its original area by day 14 . At 28 days, the wounds from both groups had achieved an average of $84 \%$ wound contracture (Fig. 7).

\section{Macroscopic evaluation}

Representative images of each group are shown in Fig. 8 . Throughout the 28 days, no signs of infection, for example, purulent discharge, were seen in wounds from the treatment and control groups. All the wounds showed a similar extent of granulation and epithelialization. Scarring was minimal for both groups at the end of the study.

We found it more difficult to handle Mepitel during application because of its strong adherence to the gloves and surgical instruments. Displacement of the dressing from the wound site was more pronounced in the treatment group. Dressings from both groups were minimally adherent to the wounds, resulting in minimal trauma during dressing change. Dressings from the treatment group were able to conform to the wound surfaces with greater ease, compared to the controls. Wounds from the treatment group showed a greater extent of exudate collection beneath the dressings, compared to the controls. Exudate collection for the treatment group spanned the surface area of the wound. Exudate collection for the controls covered less than the full surface area of the wound.

\section{Histology}

Histology was carried out using hematoxylin and eosin, and
Masson's trichrome stains. Tissue regeneration was observed from day 7 onwards in both groups (Fig. 9A, B). By day 28, the wounds were fully healed with distinct, mature layers of epidermis (Fig. 9C, D; black arrows) and dermis. The regenerated tissue was aligned with the tension axis of the skin. Both the treatment and control groups showed the presence of collagen at 7 days post-surgery. At complete healing, both groups showed well developed stratified squamous epithelium, dermis consisting of hair follicles, bundles of collagen tissue, blood vessels and nerve fibres. Both treatment and control groups showed similar progress in healing for all points in time.

\section{DISCUSSION}

Physiological and mechanical factors contribute to an ideal wound dressing. Optimal healing can be achieved by maintaining a moist wound environment to avoid desiccation, prevent infection, and reduce inflammation. A moist wound environment encourages the migration of enzymes, cytokines, growth factors, and white blood cells to the site of healing [10]. Mechanical factors include ease of handling, the presence of an avenue to allow drainage of effluent, being non-adherent to the wound bed in order to minimize trauma during removal, and the ability to conform adequately to the wound surface. An ideal wound dressing should also be able to retain its position at the wound site, and should be able to withstand sterilization processes [11]. There has yet to be a single wound dressing that is able to provide all the factors necessary for an ideal wound-healing environment.

Mepitel has most of the characteristics of an ideal wound dressing and served as the control for our experiment. It is a polyamide silicon wound dressing with extensive applications. It 
Fig. 9. Histology

(A) Wound dressed with Mepitel (black arrow) at postoperative day 7 (H\&E, $\times 100)$. (B) Wound dressed with bio-conjugated PCL membrane (black arrow) at postoperative day $7\left(H \& E_{1} \times 100\right)$. (C) Wound dressed with Mepitel (black arrow) at postoperative day $28\left(H \& E_{1} \times 100\right)$. (D) Wound dressed with bio-conjugated PCL membrane (black arrow) at postoperative day $28\left(\mathrm{H} \& \mathrm{E}_{1} \times 100\right)$. PCL, polycaprolactone.
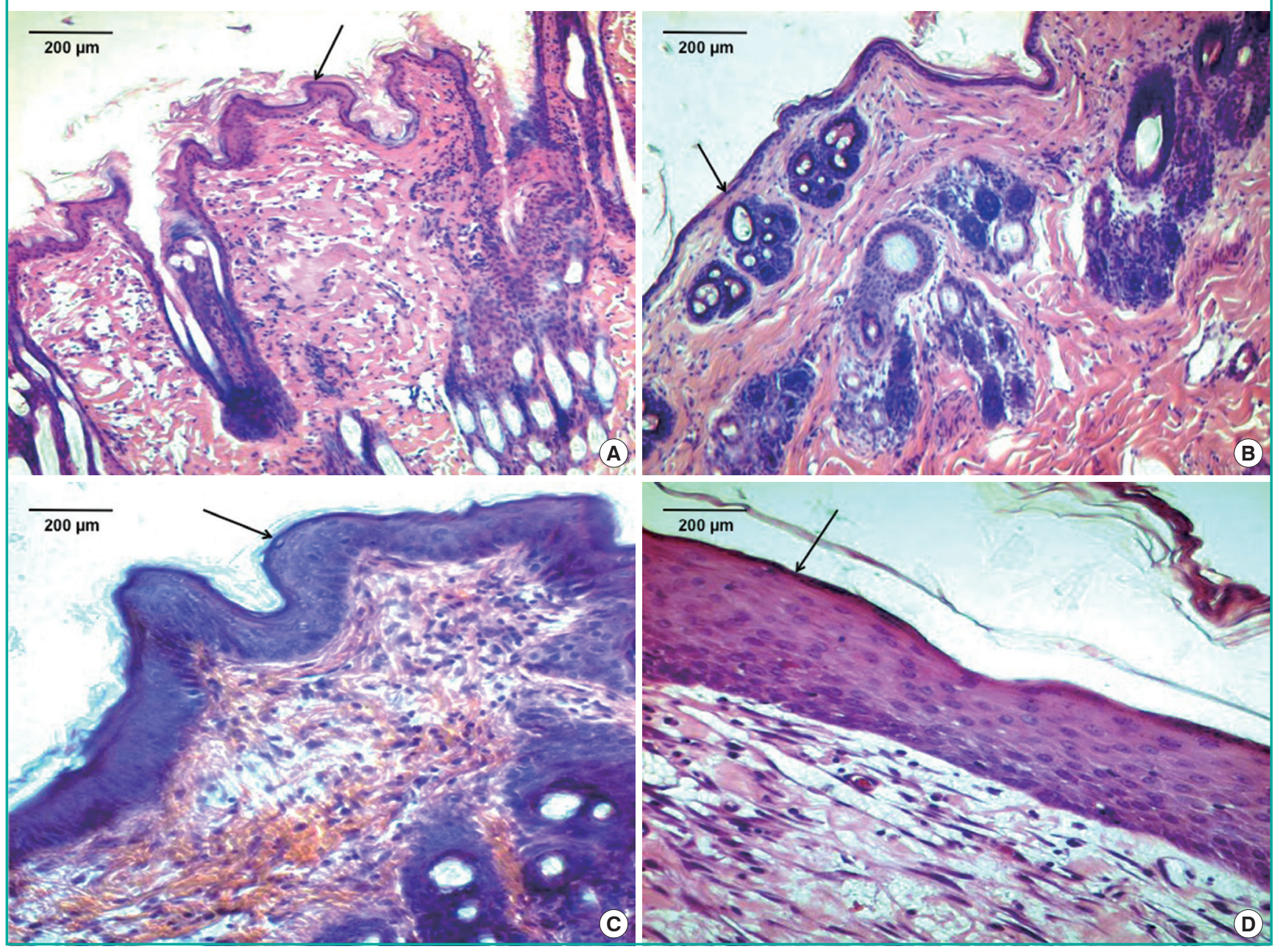

has been used in traumatic wounds, chronic wounds, burns, and fixation of skin grafts [12]. It has been shown to increase the rate of granulation and re-epithelialization. The adequate drainage of exudates from the wound bed is achieved with pores $(1.2 \mathrm{~mm}$, 14 per $\mathrm{cm}^{2}$ ) that span the dressing. It has the added advantage of being non-adherent to wounds, minimizing wound trauma and pain during dressing changes [13].

The application of Mepitel over concave surfaces such as the groin and axilla is difficult [14]. The high cost of Mepitel is also another disadvantage [14]. In our experience, Mepitel has tended to adhere strongly to gloves and surgical instruments, making it difficult to handle. Mepitel could be improved by decreasing its thickness, making it lightweight, and increasing its flexibility. This would make it more suitable for facial wounds and wounds over skin creases. Modifying its adhesive properties to prevent adherence to gloves and instruments would also be an advantage. The development of a dressing without these pitfalls while

retaining the superior characteristics of Mepitel would be ideal.

PCL has been utilized as an ultra-thin film for dressing cutaneous wounds and as a delivery vehicle for gentamicin sulphate [2]. It possesses the advantages of being stable in ambient conditions, inexpensive, and readily available [3]. PCL has been shown to possess excellent mechanical strength and has the ability to prevent contamination by microorganisms due to its thin geometry [7]. The inflammatory response, which leads to scarring, is minimized because of decreased foreign material implantation.

HyA plays a major role in facilitating wound healing. The foetal skin contains a large amount of HyA, facilitating wound healing without scar formation [15]. HyA provides a hydrophilic extra-cellular environment, which facilitates the migration of mesenchymal, epithelial, and inflammatory cells to the site of healing [16]. The autolytic debridement of the wounds is enhanced by enzymes secreted from white blood cells [17]. This prepares the wound for the repair phase. The present growth factors 
stimulate re-epithelialization and fibrous tissue deposition. The inflammatory response of wound healing is also shortened with HyA [4], allowing rapid advancement into the remodelling phase. This translates into a faster rate of healing with reduced scarring. HyA also stimulates the production of TNF-alpha and increases cellular mitosis, promoting tissue growth and angiogenesis [18].

HyA has the ability to create an ideal environment to facilitate wound healing. A major drawback is its poor mechanical strength. This can be overcome by chemical modification and cross-linking. We have successfully surface grafted HyA onto PCL using our novel surface modification technique. This has equipped HyA with enough strength to serve as a wound dressing. PCL was chosen as the carrier primarily for its excellent mechanical strength and biocompatibility. Plasma-induced surface bioconjugation enables the manipulation of the surface chemistry of membranes. This allowed PCL to be used as a scaffold for the engraftment of HyA. HyA was proven to be present on the surface of our bio-conjugated PCL membrane using the PEI method of grafting. The membrane was shown by our in-vitro study to be cytocompatible (with cell viability of more than $70 \%$ ) when evaluated with human dermal fibroblast cells.

Our study showed that a bio-conjugated PCL membrane was capable of bringing on the healing of a full-thickness wound, comparable to Mepitel. The bio-conjugated PCL membrane was biocompatible and did not elicit any local or systemic inflammatory reaction. Scarring was minimal by the end of the study. Bio-conjugated PCL also has the advantage of being nonadherent to the wound, similar to Mepitel. This would minimize trauma to the wound site and pain during dressing change.

Mepitel has its disadvantages, as stated above. We engineered the bio-conjugated PCL membrane to overcome the limitations of Mepitel. Bio-conjugated PCL exhibited a greater ease of handling compared to Mepitel. It did not stick onto gloves or surgical instruments, and did not crumple during dressing application and change. Being an ultra-thin membrane (thickness of $10-30 \mu \mathrm{m})$, it conformed better to the contours of the wounds. This would likely lead to greater ease of handling during application to a concave wound surface. It also has the advantage of being lightweight, providing greater patient comfort. A lightweight dressing should also reduce traction on the surrounding skin and reduce friction during wound healing. Being fully bioresorbable makes the bio-conjugated PCL membrane an ideal dressing for chronic wounds. It is degraded via hydrolysis over a period of 1 to 2 years, depending on the amount implanted [19]. It can be left at the wound site without removal or dressing change, minimizing trauma to the wound. Only the secondary absorbent dressing needs to be changed regularly.
Compared to Mepitel, the bio-conjugated PCL membrane also has the added advantage of retaining exudate at the wound site. Wounds treated with bio-conjugated PCL membrane showed a greater extent of exudate collection beneath the dressings, filling the surface area of the wound. Exudate from an acute wound creates a moist wound environment necessary for the diffusion of enzymes, cytokines, growth factors and the migration of cells necessary for wound healing. It also contains nutrients for cell metabolism and facilitates autolytic debridement [20].

However, excessive exudate might impede wound healing. The surrounding skin can be macerated. Physical and psychosocial morbidity could also arise. Dressings may also have to be changed more often, placing additional stress on health care resources [21]. Exudate from chronic or infected wounds would also impede wound healing. Exudate from these wounds contains proteolytic enzymes that cause the breakdown of growth factors and the surrounding skin, and also increases inflammation [20].

We have observed from this experiment that exudate from wounds dressed with bio-conjugated PCL drained from the margins into the secondary layer of gauze. The extent of exudate retention could be reduced by creating pores in the membrane. Wounds with excessive discharge will have to be dressed adequately with a secondary absorbent layer such as gauze. Desiccated exudate that had collected was removed by rubbing the area with moist cotton balls.

One of the major drawbacks of Mepitel is its cost. PCL has a relatively lower cost of production compared to other aliphatic polyesters and is widely available [22]. HyA is also readily available by means of microbial biosynthesis [23]. The cost of fabricating bio-conjugated PCL membranes as described above is low, and the fabrication process is relatively simple. This allows us to offer the market an effective yet economical dressing.

Wound infection causes significant morbidity and mortality, and is also a financial burden for hospitals. Up to $38 \%$ of hospital acquired infections are caused by wound infections [24]. A sterile dressing is necessary to prevent wound contamination and subsequent infection. Terminal sterilization and aseptic processing are possible methods for sterilizing bio-conjugated PCL membrane. Gamma irradiation and low temperature hydrogen peroxide gas are feasible methods for terminal sterilization. Steam sterilization is not recommended, as it will affect the structural integrity of PCL [25].

Future advances in bio-conjugated PCL membranes would include the incorporation of anti-inflammatory and anti-bacterial agents for infected wounds. Being light-weight and ultra-thin, it is ideal for facial wounds and wounds over skin creases, providing maximal comfort for the patient. Having the ability to retain 
moisture, it can be used for wounds with low production of exudates or desiccated wounds. HyA has been shown to reduce adhesion formation in the peritoneal cavity. In addition to being a wound dressing, bio-conjugated PCL membrane has the potential to be used as an anti-adhesion membrane following abdominal surgery.

Bio-conjugated polycaprolactone membranes are comparable to Mepitel as a safe and efficacious wound dressing. They have the factors necessary to create an optimal environment for wound healing. Compared to Mepitel, they have the added advantage of being bioresorbable, ultra-thin, and light-weight, and also have better handling qualities.

\section{REFERENCES}

1. Pontieri-Lewis V. Principles for selecting the right wound dressing. Medsurg Nurs 1999;8:267-70.

2. Teo EY, Ong SY, Chong MS, et al. Polycaprolactone-based fused deposition modeled mesh for delivery of antibacterial agents to infected wounds. Biomaterials 2011;32:279-87.

3. Hutmacher DW. Scaffolds in tissue engineering bone and cartilage. Biomaterials 2000;21:2529-43.

4. Chen WY, Abatangelo G. Functions of hyaluronan in wound repair. Wound Repair Regen 1999; 7:79-89.

5. Tiaw KS, Teoh SH, Chen R, et al. Processing methods of ultrathin poly(epsilon-caprolactone) films for tissue engineering applications. Biomacromolecules 2007;8:807-16.

6. Chong MS, Chan J, Choolani M, et al. Development of cellselective films for layered co-culturing of vascular progenitor cells. Biomaterials 2009;30:2241-51.

7. $\mathrm{Ng} \mathrm{KW}$, Achuth $\mathrm{HN}$, Moochhala S, et al. In vivo evaluation of an ultra-thin polycaprolactone film as a wound dressing. J Biomater Sci Polym Ed 2007; 18:925-38.

8. Gopinath D, Kumar MS, Selvaraj D, et al. Pexiganan-incorporated collagen matrices for infected wound-healing processes in rat. J Biomed Mater Res A 2005;73:320-31.

9. Noorjahan SE, Sastry TP. An in vivo study of hydrogels based on physiologically clotted fibrin-gelatin composites as wound-dressing materials. J Biomed Mater Res B Appl Biomater 2004;71:305-12.

10. Jones V, Harding K. Moist wound healing. In: Krasner DL, Rodeheaver GT, Sibbald RG, editors. Chronic wound care: a clinical source book for healthcare professionals. Wayne, PA: HMP Communications; 2001. p.245-52.

11. Hanna JR, Giacopelli JA. A review of wound healing and wound dressing products. J Foot Ankle Surg 1997;36:2-14.

12. White R, Morris C. Mepitel: a non-adherent wound dressing with Safetac technology. Br J Nurs 2009; 18:58-64.

13. Campanella SD, Rapley P, Ramelet AS. A randomised controlled pilot study comparing Mepitel((R)) and SurfaSoft $((\mathrm{R}))$ on paediatric donor sites treated with Recell((R)). Burns 2011;37:1334-42.

14. Deved M, Sengezer M, Kopal C. Use of Mepitel on grafted areas in burn patients. Ann Burns Fire Disasters 1999;12: 103-6.

15. Longaker MT, Chiu ES, Adzick NS, et al. Studies in fetal wound healing. V. A prolonged presence of hyaluronic acid characterizes fetal wound fluid. Ann Surg 1991;213:292-6.

16. Toole BP, Knudson CB, Munaim SI, et al. Hyaluronate-cell interactions and regulation of hyaluronate synthesis during embryonic limb development. In: Abatangelo C, editor. Cutaneous development, aging and repair. Padova: Liviana Press; 1988. p. 138-45.

17. Campbell BG. Current concepts and materials in wound bandaging. Proc North Am Vet Conf 2004;18:1217-9.

18. McCarty MF. Glucosamine for wound healing. Med Hypotheses 1996;47:273-5.

19. Hutmacher DW. Scaffold design and fabrication technologies for engineering tissues--state of the art and future perspectives. J Biomater Sci Polym Ed 2001;12:107-24.

20. White R, Cutting KF. Modern exudate management: a review of wound treatments [Internet]. World Wide Wounds; c2001 [cited 2014 Sep 17]. Available from: http://www. worldwidewounds.com/2006/september/White/ModernExudate-Mgt.html.

21. World Union of Wound Healing Societies (WUWHS). Principles of best practice: Wound exudate and the role of dressings. A consensus document. London: MEP Ltd.; 2007.

22. Woodruff MA, Hutmacher DW. The return of a forgotten polymer-Polycaprolactone in the 21st century. Prog Polym Sci 2010;35:1217-56.

23. Chong BF, Blank LM, McLaughlin R, et al. Microbial hyaluronic acid production. Appl Microbiol Biotechnol 2005; 66:341-51.

24. Ford DA, Koehler SH. A creative process for reinforcing aseptic technique practices. AORN J 2001;73:446-50.

25. Woodruff MA, Hutmacher DW. The return of a forgotten polymer-Polycaprolactone in the 21 st century. Progress in Polymer Science 2010;35:1217-56. 